\title{
ANÁLISIS COMPARATIVO DE LAS PROPIEDADES FÍSICO-MECÁNICAS DE LA MADERA DE FERNÁN SÁNCHEZ (Triplaris cumingiana Fish. Mey.) DE QUEVEDO Y BALZAR
}

\author{
Rommel Crespo Gutiérrez ${ }^{1}$, Edwin Jiménez Romero², Pedro Suatunce Cunuhay ${ }^{1}$, \\ Guillermo Law Blanco ${ }^{2}$ y Carlos Sánchez Fonseca ${ }^{1}$
}

\begin{abstract}
${ }^{1}$ División Forestal, Unidad de Investigación Cientifica y Tecnológica, Universidad Técnica Estatal de Quevedo. Av. Walter Andrade. Km 1 112 vía a Santo Domingo, C.P. 73. Quevedo, Los Ríos, Ecuador. rscgtexcmex@hotmail.com ${ }^{2}$ Escuela de Ingeniería Forestal, Facultad de Ciencias Ambientales, Universidad Técnica Estatal de Quevedo.
\end{abstract}

\section{RESUMEN}

Se estudió las propiedades físico-mecánicas de la madera de Fernán Sánchez (Triplaris cumingiana Fish. Mey.) procedente de Quevedo y Balzar. Los objetivos fueron: determinar el porcentaje de duramen y albura de los árboles, las propiedades físicas en estado verde y anhidro de la madera, las propiedades mecánicas en estado de equilibrio y las principales diferencias físico mecánicas de la madera. Se colectaron 10 árboles de Fernán Sánchez ( 5 en Quevedo y 5 en Balzar) con edades entre 13 a 18 años. De cada árbol se obtuvieron dos trozas de $2.50 \mathrm{~m}$ de largo y de cada troza se obtuvo un tablón central de $10 \mathrm{~cm}$ de ancho por $2.50 \mathrm{~m}$ de longitud del cual se extrajo las viguetas y finalmente las probetas. Se elaboraron 60 probetas (6 probetas por árbol) para los ensayos de las propiedades físicas. Para el estudio de las propiedades mecánicas se elaboraron 60 probetas (1 por cada ensayo por árbol). La metodología empleada para el estudio de las propiedades físicas y mecánicas se fundamentó en las normas ASTM D 143-94 (American Society for Testing and Materials). Los resultaron mostraron que no existe diferencia significativa entre la madera de las dos zonas. La madera de Fernán Sánchez de Quevedo y Balzar presentó mediana densidad, por lo que tiene mejores posibilidades de uso en la fabricación de muebles y gabinetes, armazón en construcciones, molduras, carpintería en general, entablados, puertas y ventanas, entre las principales.

Palabras clave: Propiedades físico-mecánicas, duramen, albura, probeta.

\section{ABSTRACT}

It was studied the physical-mechanical properties of the Fernán Sánchez wood (Triplaris cumingiana Fish. Mey.) coming from Quevedo and Balzar. The objectives were: to determine the heartwood and sapwood percentage of the trees, the physical properties in green and anhydrous state of the wood, the mechanical properties in balance state and the main physical-mechanical differences of the wood. 10 trees of Fernán Sánchez were collected (5 in Quevedo and 5 in Balzar) with ages among 13 to 18 years. Of each tree two logs of $2.50 \mathrm{~m}$ of long was obtained and of each log a central plank of $10 \mathrm{~cm}$ of wide by $2.50 \mathrm{~m}$ of longitude was obtained of which beams was extracted and finally the specimens. 60 specimens were elaborated ( 6 specimens for tree) for the rehearsals of the physical properties. For the study of the mechanical properties 60 specimens were elaborated ( 1 for each rehearsal for tree). The methodology used for the study of the physical and mechanical properties was based in the norms ASTM D 143-94 (American Society for Testing and Materials). The results showed that significant difference doesn't exist among the wood of the two areas. The wood of Fernán Sánchez of Quevedo and Balzar presented medium density, for what has better use possibilities in the production of furniture and cabinets, frame in constructions, molds, carpentry in general, boardings, doors and windows, among the main ones.

Key words: Physical-mechanical properties, heartwood, sapwood, specimens.

\section{INTRODUCCIÓN}

El Fernán Sánchez es un árbol de copa redonda, mediana de ramas huecas y anilladas en los nudos. El tronco es recto, cilíndrico y delgado, a veces con raíces tablares bajas y redondas. La corteza externa es de color gris claro, de apariencia escamosa. Se descorteza en tiras largas. La corteza interna es de color rosado claro y de sabor amargo. La madera tiene un olor y sabor ausente o no distintivo, la albura es de color marrón muy pálido con transición gradual a duramen de color rosado cremoso, con vetas características de tonos marrón grisáceo. Presenta un mediano lustre, grano recto algo

Recibido: Noviembre, 2007. Aceptado: Mayo, 2008 Publicado como ARTÍCULO en Ciencia y Tecnología 1: 79-85. 2008. entrecruzado, textura mediana, líneas vasculares visibles a simple vista y un peso y dureza medianos (Vásquez, 1983).

Esta especie maderable se encuentra distribuida desde México hasta Venezuela, Colombia, Ecuador, Perú y Bolivia. En el Ecuador se distribuye desde la costa de Esmeraldas hasta la Provincia de El Oro y centro, norte y sur oriente del país, localizándose frecuentemente en bosque explotado en altitudes de 100 a 1,000 m (Vásquez, 1983). Se desarrolla en bosque húmedo tropical y premontaño, hasta seco tropical (bh-T, bh-PM, bs-T). Generalmente crece asociada con especies como Cespedezia sphatulata, Ochroma pyramydale, Cordia alliodora, Trichospermum mexicanum, 
Cecropia garciae (Junta del Acuerdo de Cartagena, 1981).

La madera de Fernán Sánchez se la puede emplear para fabricar chapas, chapas decorativas, muebles, revestimientos, parquet, embalaje, encofrado y construcciones (Junta del Acuerdo de Cartagena, 1981). La madera es suave, pero firme, liviana, de grano recto y textura mediana, fácil de labrar y toma un buen pulimento, pero no es durable ni resistente a la podredumbre (Delgado, 1994 citado por Ibarra y Medina, 2005). Posee un Factor de Runkel de 0.34 por lo que es muy buena para la fabricación de papel (Vásquez, 1983).

La determinación de las propiedades físicas y mecánicas de la madera es un requisito indispensable para asignar los usos más adecuados a cada especie. Estos ensayos, complementados con estudios de elementos estructurales en escala normal de servicio, permiten establecer procesos industriales automatizados para la producción y clasificación en serie de los mismos elementos estructurales. Sin embargo, las investigaciones de la madera no pueden realizarse en forma arbitraria, las mismas deben obedecer a patrones previamente establecidos a nivel internacional, con el fin de poder establecer comparaciones entre las especies estudiadas por diferentes laboratorios en diferentes partes del mundo. Estos patrones de ensayo son conocidos con el nombre de Normas, las cuales son asignadas específicamente para cada material (Arroyo, 1983 citado, por Rivero, 2004).

Los ensayos sobre las propiedades físicas y mecánicas de las maderas se realizan según la norma utilizada, generalmente con el objeto de proponer los usos probables y proporcionar los datos necesarios para el cálculo de estructuras de maderas. Es necesario que los datos obtenidos permitan el uso adecuado de la madera en proporción mínima, y que garanticen seguridad en cuanto a parámetros de diseño (Hoheisel, 1981, citado por Encalada y Castillo, 1988).

El comportamiento físico de la madera está constituido por una serie de propiedades, las cuales en conjunto pueden definirse como propiedades físicas de la madera. Partiendo de esta concepción, las propiedades físicas de la madera son el conjunto de propiedades que caracterizan el comportamiento físico de la misma (Arroyo, 1983 citado por Rivero, 2004).

Las propiedades mecánicas de la madera son la expresión de su comportamiento bajo la aplicación de fuerzas o cargas. Este comportamiento puede sufrir varias modificaciones, dependiendo del tipo de fuerza aplicada y de las diferencias básicas en la organización estructural de la madera (Pérez,1983, citado por Encalada y Castillo, 1988). El conocimiento de las propiedades mecánicas de la madera proporciona los datos básicos requeridos por la ingeniería en el cálculo y diseño para la construcción y otros usos (Pérez, 1983; Alcántara, s.f. citados por Jaramillo y Ontaneda, 1989).

El estudio de las propiedades mecánicas conjuntamente con las propiedades físicas constituyen una base objetiva fundamental para determinar los posibles usos a que puede ser destinada una madera. Para lograr este objetivo, los ensayos deben ejecutarse en muestras pequeñas de maderas libres de defectos y de acuerdo a normas establecidas internacionalmente (Tuset y Duran, 1974, citados por Encalada y Castillo, 1988).

En resumen se puede decir que la selección y utilización de la madera de una determinada especie con fines industriales, sólo podrán ser realizadas con el conocimiento preciso de las cualidades tanto físicas como mecánicas de la madera (Serrano et al., 2002, citados por Rivero, 2004).

\section{MATERIALES Y MÉTODOS}

La investigación se realizó en la zona de Quevedo ubicada a $73 \mathrm{msnm}$, con una precipitación anual de $2,355.38 \mathrm{~mm}$, temperatura promedio anual de $25.58^{\circ} \mathrm{C}$, humedad relativa de $85.93 \%$, heliofanía media anual de 879.42 horas luz y tipo de suelo franco limoso con un $\mathrm{pH}$ de 6.5-7.0. La zona de Balzar se encuentra a 40 msnm, presenta una precipitación media anual de 1,222 $\mathrm{mm}$, temperatura promedio anual de $24.4{ }^{\circ} \mathrm{C}$, humedad relativa de $72.9 \%$, heliofanía media anual de 881 horas luz y tipo de suelo arcilloso-arenoso con un pH 5.9-7.1.

Se eligieron al azar 5 árboles en Quevedo y 5 árboles en Balzar, los cuales fueron apeados utilizando una motosierra. Estos árboles presentaron buenas características fitosanitarias, fustes rectos, cilíndricos y fueron representativos tanto en diámetro como en altura. Los datos de los árboles en pie y apeados fueron registrados en una hoja de campo.

El cálculo de la cantidad aproximada de duramen y albura existentes en cada una de las trozas, de las cuales se extrajo las probetas para los diferentes ensayos, se efectuó mediante una relación entre el volumen total y el volumen de duramen, aplicando las siguientes fórmulas:

$$
\begin{gathered}
\text { Volumen total }\left(m^{3}\right)=0.7854 * \text { largo } *\left(\frac{\text { Diámetro mayor }+ \text { diámetro menor }}{2}\right)^{2} \\
\text { Volumen albura }=\text { volumen total }- \text { volumen duramen } \\
\text { Albura\% }=\frac{\text { volumen albura } * 100}{\text { volumen total }} \\
\text { Duramen } \%=100-\text { albura\% }
\end{gathered}
$$

Una vez apeado se extrajeron dos trozas de cada árbol y de cada una de ellas se obtuvo un tablón central 
de $10 \mathrm{~cm}$ de ancho por $2.50 \mathrm{~m}$ de largo, los cuales fueron marcados para su posterior identificación. De cada tablón se obtuvieron viguetas de $10 \times 10 \mathrm{~cm}$ de ancho por $250 \mathrm{~cm}$ de largo. De una de estas viguetas utilizando canteadora, sierra circular, cepilladora y lijadora se elaboraron 60 probetas para el ensayo de las propiedades físicas de acuerdo a las normas ASTM (1996) y teniendo en cuenta la correcta orientación de las caras. Puesto que los árboles fueron apeados varias semanas antes, las probetas fueron sumergidas en agua durante 24 horas para que adquirieran suficiente cantidad de agua y que el contenido de humedad sea mayor al $30 \%$. En cada una de las probetas se determinó su peso, volumen y dimensiones en el estado verde y seco al horno, para lo cual se utilizó una balanza digital, un calibrador y una estufa eléctrica.

Las propiedades físicas contenido de humedad en estado verde y anhidro, densidad, peso específico básico y contracción fueron determinadas por medio del secado en estufa eléctrica (diferencia de peso), provista de un termorregulador en el Laboratorio de Biotecnología de la Universidad Técnica Estatal de Quevedo. Se aplicó un calentamiento gradual $\left(45^{\circ} \mathrm{C}, 75^{\circ} \mathrm{C}\right.$ y $\left.103+2{ }^{\circ} \mathrm{C}\right)$ hasta que las probetas alcanzaron un peso constante. Se utilizaron las siguientes fórmulas:

$$
\begin{gathered}
\text { Contenido humedad verde }(\%)=\frac{\text { Peso húmedo }- \text { Peso seco }}{\text { Peso seco }} \times 100 \\
\text { Contenido humedad anhidro }(\%)=\frac{\text { Peso húmedo }- \text { Peso seco }}{\text { Peso húmedo }} \times 100 \\
\text { Densidad verde }\left(g \mathrm{~cm}^{3}\right)=\frac{\text { Peso verde }}{\text { Volumen verde }} \\
\text { Pensidad anhidra }\left(g \mathrm{~cm}^{3}\right)=\frac{\text { Peso seco al horno }}{\text { Volumen seco al horno }} \\
\text { Contracción tangencial total }(\%)=\frac{\text { Peso seco basíco }=\frac{\text { Volumen húmedo } / \text { densidad del agua }}{\text { Dtv }} \text {. } 100}{}
\end{gathered}
$$

Donde:

$D t v=$ dimensión tangencial en estado verde

$D t a=$ dimensión tangencial en estado anhidro

$$
\text { Contracción radial total }(\%)=\frac{\text { Drv }- \text { Dra }}{\text { Drv }} \times 100
$$

Donde:

$D r v=$ dimensión radial en estado verde

Dra $=$ dimensión radial en estado anhidro
Contracción longitudinal total $(\%)=\frac{D l v-D l a}{D l v} \times 100$

Donde:

$D l v=$ dimensión longitudinal en estado verde

$D l a=$ dimensión longitudinal en estado anhidro

Contracción volumétrica total $=C t t+C r t+C l t$

Donde:

$C t t=$ Contracción tangencial total

$C r t=$ Contracción radial total

$C l t=$ Contracción longitudinal total

$$
\text { Relación } T / R=\frac{\text { Contracción Tangencial \% }}{\text { Contracción radial \% }}
$$

Las 60 probetas para el ensayo de las propiedades mecánicas (1 probeta por árbol, 10 por ensayo) fueron elaboradas de las viguetas, con el contenido de humedad en estado de equilibrio, en el taller de la madera del Laboratorio de Ensayos de Materiales y Modelos de la Universidad Central del Ecuador en la ciudad de Quito, de acuerdo con las normas ASTM (Annual Book of ASTM Andards, 1996), y fueron sometidas a los ensayos de: flexión estática, compresión paralela y perpendicular a la fibra, dureza Brinnell de extremos y lados, corte paralelo a la fibra y extracción de clavos en las tres caras. Para ello se utilizó la maquina universal de ensayos Tinius Olsen con sus respectivos accesorios.Se realizaron los siguientes cálculos:

\section{Flexión estática}

$$
\begin{aligned}
& \text { Módulo } \\
& \text { de ruptura }\left(\mathrm{kg} \mathrm{cm}^{2}\right)=\frac{1.5 \times \text { carga máxima } \times \text { luz entre apoyos }}{\text { Ancho de probeta } \times \text { (altura probeta) })^{2}} \\
& \begin{array}{c}
\text { Módulo de } \\
\text { elasticidad }\left(\mathrm{kg} \mathrm{cm}^{2}\right)=\frac{0.25 \times(\text { luz entre apoyos) })^{3} \times C L P}{\text { Ancho de probeta } \times \text { (altura probeta) })^{3} \times L P} \\
\text { Donde: } \\
C L P=\text { Carga en el límite proporcional }(\mathrm{kg}) \\
D L P=\text { Deformación en el límite proporcional }(\mathrm{cm})
\end{array}
\end{aligned}
$$

\section{Compresión paralela a la fibra}

$$
\underset{\text { Módulo }}{\text { de ruptura }\left(\mathrm{kg} \mathrm{cm}^{2}\right)}=\frac{\text { Carga máxima }}{\text { Ancho de probeta x espesor de probeta }}
$$

\section{Compresión perpendicular a la fibra}

$E L P\left(\mathrm{~kg} \mathrm{~cm}^{2}\right)=\frac{\text { Carga en el límite proporcional }}{\text { Ancho de pieza metálica x espesor de pieza metálica }}$ 
Donde:

$E L P=$ Esfuerzo en el límite proporcional

\section{Corte paralelo a la fibra}

$$
\text { Esfuerzo máximo }\left(\mathrm{kg} \mathrm{cm}^{2}\right)=\frac{\text { Carga máxima }(\mathrm{kg})}{5 \mathrm{~cm} \times 5 \mathrm{~cm}}
$$

Los datos para los ensayos de Dureza Brinnell y de Extracción de clavos se obtuvieron directamente de la máquina, sin necesidad de hacer ningún cálculo adicional. Se realizó un análisis de comparación de medias a través de la prueba de " $t$ " a un nivel de significancia de 0.10 .

\section{RESULTADOS}

\section{Porcentaje de duramen y albura}

Las medias porcentuales de duramen y albura de las 10 trozas de Fernán Sánchez procedentes de Quevedo y 10 trozas de Fernán Sánchez procedentes de Balzar presentaron diferencias significativas (Cuadro 1).

\section{Propiedades físicas}

El contenido de humedad anhidro y la densidad en estado verde presentaron diferencias significativas; las demás variables no presentaron diferencias significativas (Cuadro 2). La madera de Fernán Sánchez de Quevedo y Balzar se clasificó como de mediana densidad en estado anhidro, bajo peso específico, baja contracción volumétrica total y de una relación Tangencial/Radial (T/R) moderadamente estable para la madera de Fernán Sánchez de Quevedo y estable para la madera de Fernán Sánchez de Balzar (Cuadro 3).

\section{Propiedades mecánicas}

El ensayo de dureza Brinnell en la cara transversal presentó diferencias significativas, los demás ensayos de las propiedades mecánicas de la madera de Fernán Sánchez de las zonas de Quevedo y Balzar no presentaron diferencias significativas (Cuadro 4).

El MOR y el MOE para la madera de Fernán Sánchez de Quevedo y Balzar en la flexión estática fueron bajos. El MOR en la compresión paralela a la fibra y el ELP en la compresión perpendicular a la fibra fueron bajos en las maderas de ambas zonas. La madera de Fernán Sánchez de las dos zonas, en las caras transversal, radial y tangencial, se clasificó como baja o blanda, de acuerdo a la dureza Brinnell. El MOR en el corte paralelo a la fibra en la cara radial fue bajo en la madera de Fernán Sánchez de Quevedo y mediano en la madera de Fernán Sánchez de Balzar. La madera de Fernán Sánchez de ambas zonas presentó una resistencia a la extracción de clavos mediana en la cara transversal, alta en la cara radial y en la cara tangencial en Quevedo muy alta y en Balzar alta (Cuadro 5).

Cuadro 1. Porcentajes de duramen y albura en trozas de Fernán Sánchez procedente de las zonas de Balzar y Quevedo

\begin{tabular}{cccc}
\hline \multicolumn{2}{c}{ Duramen (\%) } & \multicolumn{2}{c}{ Albura (\%) } \\
\hline Balzar & Quevedo & Balzar & Quevedo \\
\hline $71.66 \mathrm{a}^{\dagger}$ & $67.40 \mathrm{~b}$ & $28.34 \mathrm{~b}$ & $32.60 \mathrm{a}$ \\
\hline
\end{tabular}

${ }^{\dagger}$ Promedios con letras iguales entre columnas, no difieren entre si $(\mathrm{p} \geq 0.10)$.

Cuadro 2. Valores promedios de las propiedades físicas de la madera de Fernán Sánchez de Quevedo y Balzar

\begin{tabular}{lrr}
\hline \multirow{2}{*}{ Ensayos } & \multicolumn{2}{c}{ Localidad } \\
\cline { 2 - 3 } & Quevedo & Balzar \\
\hline Contenido de humedad verde (\%) & $87.81 \mathrm{a}^{\dagger}$ & $93.46 \mathrm{a}$ \\
Contenido de humedad anhidra $(\%)$ & $46.31 \mathrm{a}$ & $42.30 \mathrm{~b}$ \\
Densidad estado verde $\left(\mathrm{g} \mathrm{cm}^{3}\right)$ & $0.80 \mathrm{a}$ & $0.75 \mathrm{~b}$ \\
Densidad estado anhidro $\left(\mathrm{g} \mathrm{cm}^{3}\right)$ & $0.47 \mathrm{a}$ & $0.48 \mathrm{a}$ \\
Peso específico básico* & $0.43 \mathrm{a}$ & $0.43 \mathrm{a}$ \\
Contracción total radial (\%) & $3.60 \mathrm{a}$ & $3.96 \mathrm{a}$ \\
Contracción total tangencial (\%) & $5.43 \mathrm{a}$ & $5.27 \mathrm{a}$ \\
Contracción total longitudinal (\%) & $0.38 \mathrm{a}$ & $0.37 \mathrm{a}$ \\
Contracción total volumétrica (\%) & $9.41 \mathrm{a}$ & $9.60 \mathrm{a}$ \\
Relación tangencial/radial & $2.06 \mathrm{a}$ & $1.73 \mathrm{a}$ \\
\hline
\end{tabular}

*Valor adimensional.

†Promedios con letras distintas entre columnas, difieren entre si $(\mathrm{p} \geq 0.10)$. 
Cuadro 3. Categorización de la madera de Fernán Sánchez para la zona de Quevedo y Balzar según los resultados obtenidos en los ensayos de las propiedades físicas

\begin{tabular}{lcc}
\hline \multirow{2}{*}{ Propiedades } & \multicolumn{2}{c}{ Localidad } \\
\cline { 2 - 3 } & Quevedo & Balzar \\
\cline { 2 - 3 } & Mediana & Mediana \\
\hline Densidad en estado anhidra $\left(\mathrm{g} \mathrm{cm}^{3}\right)$ & Bajo & Bajo \\
Peso específico básico & Baja & Baja \\
Contracción volumétrica total $(\%)$ & Estable \\
Relación contracción tangencial/radial (T/R) & Moderadamente estable & E \\
\hline
\end{tabular}

Cuadro 4. Valores promedios de las propiedades mecánicas de la madera de Fernán Sánchez de Quevedo y Balzar

\begin{tabular}{|c|c|c|}
\hline \multirow{2}{*}{ Propiedades } & \multicolumn{2}{|c|}{ Localidad } \\
\hline & Quevedo & Balzar \\
\hline \multicolumn{3}{|l|}{ Flexión Estática } \\
\hline Módulo de ruptura (MOR, $\mathrm{kg} \mathrm{cm}^{2}$ ) & $562.94 \mathrm{a}^{\dagger}$ & $562.12 \mathrm{a}$ \\
\hline Módulo de elasticidad (MOE, $\mathrm{kg} \mathrm{cm}^{2}$ ) & $86,169.48 \mathrm{a}$ & $92,471.15 \mathrm{a}$ \\
\hline \multicolumn{3}{|l|}{ Compresión paralela a la Fibra } \\
\hline Módulo de ruptura (MOR, $\mathrm{kg} \mathrm{cm}^{2}$ ) & $347.65 \mathrm{a}$ & $390.25 \mathrm{a}$ \\
\hline \multicolumn{3}{|l|}{ Compresión perpendicular a la fibra } \\
\hline Esfuerzo al límite proporcional (ELP, $\mathrm{kg} \mathrm{cm}^{2}$ ) & $52.95 \mathrm{a}$ & $64.69 \mathrm{a}$ \\
\hline \multicolumn{3}{|l|}{ Corte paralelo a la fibra cara radial } \\
\hline Módulo de ruptura (MOR, $\mathrm{kg} \mathrm{cm}^{2}$ ) & $82.39 \mathrm{a}$ & $101.46 \mathrm{a}$ \\
\hline \multicolumn{3}{|l|}{ Extracción de clavos } \\
\hline Cara radial (carga máxima, kg) & $63.50 \mathrm{a}$ & $66.00 \mathrm{a}$ \\
\hline Cara tangencial (carga máxima, kg) & $72.00 \mathrm{a}$ & $65.00 \mathrm{a}$ \\
\hline Cara Transversal (carga máxima, kg) & $35.50 \mathrm{a}$ & $49.50 \mathrm{a}$ \\
\hline \multicolumn{3}{|l|}{ Dureza Brinnell } \\
\hline Cara radial (carga máxima, kg) & $300.00 \mathrm{a}$ & $305.50 \mathrm{a}$ \\
\hline Cara tangencial (carga máxima, $\mathrm{kg}$ ) & $285.50 \mathrm{a}$ & $360.00 \mathrm{a}$ \\
\hline Cara transversal (carga máxima, $\mathrm{kg}$ ) & $343.00 \mathrm{~b}$ & $417.00 \mathrm{a}$ \\
\hline
\end{tabular}

Cuadro 5. Categorización de la madera de Fernán Sánchez para la zona de Quevedo y Balzar según los resultados obtenidos en los ensayos de las propiedades mecánicas

\begin{tabular}{|c|c|c|}
\hline \multirow{3}{*}{ Propiedades } & \multicolumn{2}{|c|}{ Localidad } \\
\hline & Quevedo & Balzar \\
\hline & \multicolumn{2}{|c|}{ Clasificación } \\
\hline Flexión estática MOR $\left(\mathrm{kg} \mathrm{cm}^{2}\right)$ & Bajo & Bajo \\
\hline Flexión estática $\mathrm{MOE}\left(\mathrm{kg} \mathrm{cm}^{2}\right)$ & Bajo & Bajo \\
\hline Compresión paralela a la fibra MOR $\left(\mathrm{kg} \mathrm{cm}^{2}\right)$ & Bajo & Bajo \\
\hline Compresión perpendicular a la fibra ELP $\left(\mathrm{kg} \mathrm{cm}^{2}\right)$ & Bajo & Bajo \\
\hline Dureza Brinnell cara transversal (kg) & Baja o blanda & Baja o blanda \\
\hline Dureza Brinnell cara radial $(\mathrm{kg})$ & Baja o blanda & Baja o blanda \\
\hline Dureza Brinnell cara tangencial $(\mathrm{kg})$ & Baja o blanda & Baja o blanda \\
\hline Corte pararelo a la fibra cara radial MOR $\left(\mathrm{kg} \mathrm{cm}^{2}\right)$ & Bajo & Mediano \\
\hline Extracción de clavos cara transversal $(\mathrm{kg})$ & Mediana & Mediana \\
\hline Extracción de clavos cara radial $(\mathrm{kg})$ & Alta & Alta \\
\hline Extracción de clavos cara tangencial $(\mathrm{kg})$ & Muy alta & Alta \\
\hline
\end{tabular}




\section{DISCUSION}

El contenido de humedad en estado verde de la madera de Fernán Sánchez de Quevedo (87.81\%) es similar al encontrado por Martínez (1989) en un estudio en madera de Fernán Sánchez realizado en Conocoto, Ecuador, donde obtuvo un valor de $83.6 \%$. La madera de Fernán Sánchez en la zona de Quevedo y Balzar presentó un peso específico básico de 0.43 , este resultado difiere al encontrado por Martínez (1989) en un estudio realizado en madera de Fernán Sánchez en Conocoto, Ecuador donde determinó un peso específico básico de 0.53. Igualmente en un estudio realizado por Orbe (1986) en Santo Domingo de los Colorados, Ecuador, en madera de Fernán Sánchez obtuvo un valor de peso específico básico de 0.54. El mayor contenido de humedad registrado en la madera de Fernán Sánchez de Quevedo y de la zona de estudio de Martínez se debe a que estas zonas presentan una mayor precipitación que la de Balzar, por lo que el tejido leñoso de los árboles de estas zonas disponen de mayor cantidad del recurso agua para realizar sus actividades fisiológicas.

La madera de Fernán Sánchez de la zona de Quevedo presentó una densidad en estado verde de 0.80 $\mathrm{g} \mathrm{cm}^{3}$ y una densidad Anhidra de $0.47 \mathrm{~g} \mathrm{~cm}^{3}$; en la zona de Balzar presentó un valor para la densidad verde de $0.75 \mathrm{~g} \mathrm{~cm}^{3}$ y para la densidad anhidra de $0.48 \mathrm{~g} \mathrm{~cm}^{3}$, estos resultados no concuerdan con los reportados por Martínez (1989) el cual determinó para esta madera una densidad verde de $0.97 \mathrm{~g} \mathrm{~cm}^{3}$ y una densidad Anhidra de $0.60 \mathrm{~g} \mathrm{~cm}^{3}$. La madera de Fernán Sánchez estudiada por este investigador provino de un lugar más húmedo que el de las dos zonas estudiadas en esta investigación, lo que explica el porque obtuvo mayores valores tanto para la densidad verde como anhidra en la madera de esta especie.

El valor de contracción volumétrica total en la zona de Quevedo para la madera de Fernán Sánchez fue de $9.41 \%$ mientras que en Balzar presentó un valor de $9.60 \%$, los valores obtenidos no concuerdan con los presentados por Martínez (1989) quien obtuvo un valor de $11.9 \%$ de contracción volumétrica total para la madera de Fernán Sánchez. La madera de Fernán Sánchez estudiada por Martínez después del secado presentó mayor contracción volumétrica total debido a que presentaba mayor porosidad que las de las zonas de Quevedo y Balzar.

La relación de la contracción tangencial/radial total (T/R) obtenida en la madera de Fernán Sánchez de Quevedo fue de $2.06 \%$ y para la madera de Balzar de $1.73 \%$, estos resultados concuerdan con los determinados por Martínez (1989) quien obtuvo un valor de $1.8 \%$ para la relación de contracción tangencial/radial total (T/R).
Los valores de 562.94 y de $562.12 \mathrm{~kg} \mathrm{~cm}^{2}$ del módulo de ruptura (MOR) y de $86,169.48 \mathrm{~kg} \mathrm{~cm}^{2}$ y $92,471.15 \mathrm{~kg} \mathrm{~cm}^{2}$ para el módulo de elasticidad (MOE) determinados en el ensayo de flexión estática en la madera de Fernán Sánchez de la zona de Quevedo y Balzar respectivamente, difieren con los obtenidos por Martínez (1989) quien determinó un MOR de $1,019 \mathrm{~kg} \mathrm{~cm}{ }^{2}$ y un MOE de 127,000 $\mathrm{kg} \mathrm{cm}^{2}$ en un estudio realizado en madera de Fernán Sánchez en Conocoto, Ecuador.

Los valores del módulo de ruptura (MOR) obtenidos en el ensayo de compresión paralela a la fibra en la madera de Fernán Sánchez de la zona de Quevedo de 347.65 y de $390.25 \mathrm{~kg} \mathrm{~cm}^{2}$ en la zona de Balzar, no concuerdan con los obtenidos por Martínez (1989) quien obtuvo un valor de $516 \mathrm{~kg} \mathrm{~cm}^{2}$.

Los valores del esfuerzo al límite proporcional (ELP) obtenidos en el ensayo de compresión perpendicular a la fibra en la madera de Fernán Sánchez de la zona de Quevedo de $52.95 \mathrm{~kg} \mathrm{~cm}^{2}$ y de la zona de Balzar de $64.69 \mathrm{~kg} \mathrm{~cm}^{2}$ concuerdan en parte con el obtenido por Martínez (1989) quien un valor de ELP de $62 \mathrm{~kg} \mathrm{~cm}^{2}$.

El valor del módulo de ruptura (MOR) en el ensayo de corte paralelo a la fibra en la cara radial de la madera de Fernán Sánchez para la zona de Quevedo de $82.39 \mathrm{~kg} \mathrm{~cm}^{2}$ y para la zona de Balzar de $101.46 \mathrm{~kg} \mathrm{~cm}^{2}$, concuerdan en parte con el determinado por Martínez (1989) con un valor de $108 \mathrm{~kg} \mathrm{~cm}^{2}$.

Los valores de la carga máxima determinados en el ensayo de dureza Brinnell en la madera de Fernán Sánchez en la cara radial en la zona de Quevedo de $300.00 \mathrm{~kg}$ y en la zona de Balzar de $305.50 \mathrm{~kg}$; en la cara tangencial para la madera de Quevedo de 285.50 y para madera de Balzar de 360.00; y en la cara transversal en la madera de Quevedo de 343.00 y en la madera de Balzar de 417.00, difieren con los obtenidos por Martínez (1989), quien determinó un valor de carga máxima de $484 \mathrm{~kg}$ para los lados (cara radial y tangencial) y un valor de $667 \mathrm{~kg}$ para los extremos (cara transversal).

Las diferencias entre los resultados obtenidos en el presente estudio con los determinados por Martínez se deben posiblemente a la diferencia en edad, condiciones de crecimiento y sitio, altura, gradientes de humedad, dirección o desviación de las fibras y presencia de nudos. Debido a que cada árbol es distinto del resto y aun dentro de un mismo individuo las características de la madera varían con la posición en relación al eje y altura sobre el suelo.

\section{CONCLUSIONES}

El mayor contenido de humedad, en estado verde y seco al horno, fue para el Fernán Sánchez de la zona de Quevedo. La densidad de la madera de Fernán Sánchez, en estado verde fue mayor en Quevedo. 
El peso específico básico de la madera de Fernán Sánchez fue igual para ambas zonas. La contracción radial total y volumétrica total de la madera de Fernán Sánchez fueron mayores en la zona de Balzar.

La contracción tangencial total y longitudinal total fueron mayores en madera de Fernán Sánchez de Quevedo. La madera de Fernán Sánchez presentó una relación tangencial/radial (T/R) moderadamente estable en la zona de Balzar, y estable en la zona de Quevedo.

La carga $(\mathrm{kg})$ en la dureza Brinnell en la cara transversal fue mayor en la madera de Fernán Sánchez de Balzar. El módulo de ruptura (MOR) en el corte paralelo a la fibra, en la cara radial, fue mayor en la zona de Balzar.

En general, la madera de Fernán Sánchez de Balzar presentó mejores propiedades físicas y mecánicas que la de Quevedo, por cuanto las condiciones ambientales son diferentes.

\section{LITERATURA CITADA}

ASTM (American Society for Testing and Materials). 1996 Annual Book of ASTM Andards. Section 4 Construction. Volume 04.10 Wood. Pennsylvania, United States. P. 23-39, 556-559.

Encalada, O. y Castillo, F. 1988. Propiedades físicas y mecánicas de diez especies forestales de la reserva forestal "La Zarza" en la Provincia de Zamora Chinchipe. Tesis Ing. For. Loja, Ecuador. Universidad Nacional de Loja. Facultad de Ciencias Agrícolas. Escuela de Ingeniería Forestal. 132 p.

Ibarra, R. y Medina, S. 2005. Dinámica de crecimiento de cuatro especies forestales en asociación con Theobroma cacao L. en el Cantón La Maná - Cotopaxi (Segunda Fase). Tesis. Ing. For. Quevedo, Ecuador. Universidad Técnica Estatal de Quevedo. Facultad de Ciencias Ambientales. Escuela de Ingeniería Forestal. p. 16.

Jaramillo, F. y Ontaneda, G. 1989. Estudio de las características tecnológicas, trabajabilidad y usos de la especie forestal Pollalesta karstenii, Pigüe. Tesis. Ing. For. Loja, Ecuador. Universidad Nacional de Loja. Facultad de Ciencias Agrícolas. Escuela de Ingeniería Forestal. 142 p.

Junta del Acuerdo de Cartagena. 1981. Descripción general y anatómica de 105 maderas del Grupo Andino. PADT, REFORT, JUNAC. Proyectos Andinos de desarrollo tecnológico en el área de los recursos forestales tropicales. Lima, Perú. p. 209. $441 \mathrm{p}$.

Martínez, N. 1989. Tablas de propiedades FísicoMecánicas de 50 especies maderables ecuatorianas. Conocoto, Ecuador. Ministerio de Agricultura y Ganadería. Dirección Nacional Forestal. Centro de Investigación y Capacitación Forestal "Luciano Andrade Marín”. p. 7, 11, 30.

Orbe, J. 1986. Resumen de un estudio de durabilidad natural de varias maderas ecuatorianas. Conocoto, Ecuador. Ministerio de Agricultura y Ganadería. Programa Nacional Forestal. Laboratorio de Tecnología de la Madera. Centro de Investigación Forestal "Luciano Andrade Marín”. 14 p.

Rivero, J. 2004 Propiedades Físico-Mecánicas de Gmelina arbórea Roxb. y Tectona grandis Linn. F. (en línea). Bo. Metodología. Consultado el 28 jun del 2005. Disponible en http://www.monografias. com/trabajos 16/gmelina-arborea/gmelinaarborea.shtml\#top.

Vásquez, E. 1983. Descripción General Botánica y Anatómica de 52 Maderas del Ecuador. Ministerio de Agricultura y Ganadería. Programa Nacional Forestal. Centro de Capacitación e Investigación Forestal Luciano Andrade Marín. IF N ${ }^{0} 15$. p. 80 -82 . 